PLOS Computational Biology would like to thank all those who reviewed on behalf of the journal in 2014:

\begin{tabular}{|c|c|}
\hline Scott Aaronson & Philipp Altrock \\
\hline Larry Abbott & Mario Alvarez \\
\hline Pia Abel zur Wiesch & Rommie Amaro \\
\hline Maria Abou Chakra & Roee Amit \\
\hline Laith Abu-Raddad & Greg Amoutzias \\
\hline Luigi Acerbi & Eva Amsen \\
\hline Pablo Achard & Gary An \\
\hline Achilleas Achilleos & Sophia Ananiadou \\
\hline Jörg Ackermann & John Anderson \\
\hline Daniel Acuna & Bart Anderson \\
\hline Christoph Adami & Alexander Anderson \\
\hline Boris Adryan & Paul Anderson \\
\hline Stein Aerts & Marti Anderson \\
\hline Pankaj Agarwal & Ole Andreassen \\
\hline Amit Aggarwal & R. Andrew \\
\hline Matthew Agler & Steven Andrews \\
\hline Smita Agrawal & Ioan Andricioaei \\
\hline Cendra Agulhon & Rustom Antia \\
\hline Amir Aharoni & Shunsuke Aoki \\
\hline Yashar Ahmadian & Kazuhiro Aoki \\
\hline Alaa Ahmed & Juan Aparicio \\
\hline Yong-Yeol Ahn & Udayan Apte \\
\hline Sunil Ahuja & Johan Åqvist \\
\hline Christopher Aiken & Salva Ardid \\
\hline Elizabeth Ailes & Fernando Arenzana-Seisdedos \\
\hline Antti Airola & Jacob Arguello \\
\hline Edoardo Airoldi & Nimalan Arinaminpathy \\
\hline Laurence Aitchison & Stephane Aris-Brosou \\
\hline Thomas Akam & Anton Arkhipov \\
\hline Joshua Akey & Roger Armen \\
\hline Eyal Akiva & Alain Arneodo \\
\hline Sahar Akram & Manimozhiyan Arumugam \\
\hline Athena Akrami & Vinayagam Arunachalam \\
\hline Aleksei Aksimentiev & Lars Arvestad \\
\hline Tomas Alarcon & Hugues Aschard \\
\hline Larissa Albantakis & Giorgio Ascoli \\
\hline John Albeck & Roman Ashauer \\
\hline Réka Albert & Gregory Ashby \\
\hline Martí Aldea & Michael Assaf \\
\hline Bree Aldridge & Anand Asthagiri \\
\hline Max Alekseyev & Lauren Atlas \\
\hline Helen Alexander & Benjamin Audit \\
\hline Boian Alexandrov & Yurii Aulchenko \\
\hline Emil Alexov & Kari Auranen \\
\hline Samuel Alizon & Erik Aurell \\
\hline Yannick Allanore & Bruno Averbeck \\
\hline Patrick Aloy & Ahmet Ay \\
\hline Jeff Alstott & Nihat Ay \\
\hline Grégoire Altan-Bonnet & Sandro Azaele \\
\hline Christian Althaus & Rony Azouz \\
\hline Russ Altman & Eugenio Azpeitia \\
\hline
\end{tabular}


Madan Babu

Nicolas Bacaer

Brian Bachmann

Roland Baddeley

Gary Bader

Steven Baer

Vineet Bafna

Ivet Bahar

Wadie Bahou

Bahador Bahrami

Jane Bai

Lu Bai

Timothy Bailey

Chris Bailey-Kellogg

James Baish

Chris Bakal

Matthew Baker

Brian Baker

Ruth Baker

Chris Baker

Gabor Balazsi

Vincent Balter

Nilesh Banavali

Leah Band

Sourav Bandyopadhyay

Julio Banga

Edward Banigan

Claudia Bank

Shweta Bansal

Mukul Bansal

Svetlana Baoukina

Omri Barak

Yael Baran

Virginia Barbour

Alessandro Barducci

Lee Bardwell

Stephen Barigye

Debashis Barik

Ziv Bar-Joseph

Ernest Barreto

Adam Barrett

Xavier Barril

Simon Barthelme

Ed Bartlett

Frederic Bartumeus

Anastasia Baryshnikova

Patricia Bassereau

Philippe Bastiaens

Ugo Bastolla

Alex Bateman

Paul Bates

Mark Bathe

Francesco Battaglia

Alexis Battle

J. Fernando Bazan
Jason Bazil

Georgii Bazykin

Martin Beck

Jeff Beck

Oliver Beckstein

Attila Becskei

Michael Beer

Randy Beer

Niko Beerenwinkel

John Beggs

Chase Beisel

Alexander Beliaev

Pascal Belin

George Bell

Nicola Bellomo

Joost Beltman

Pedro Beltrao

Jan Benda

Robert Bender

Philip Benfey

Craig Benham

Asa Ben-Hur

Yoav Benjamini

Matthew Bennett

Richard Benninger

Sliman Bensmaia

Katie Bentley

Philipp Berens

Igor Berezovsky

Howard Berg

Bonnie Berger

Joshua Berke

Ari Berkowitz

Samuel Bernard

Marti Bernardo-Faura

Monica Berrondo

Anna Bershteyn

Alex Best

Robert Best

Matthias Bethge

Andreas Beyer

Pratik Bhagunde

Upinder Bhalla

Vikas Bhandawat

Nita Bharti

Sudin Bhattacharya

Tanmoy Bhattacharya

Joseph P. Bielawksi

Elaine Bignell

Ewan Birney

Inanc Birol

David Biron

Marc Birtwistle

Prakash Bisen

Jennifer Bizley 
Nils Blüthgen

Jeffrey Blanchard

Romain Blanc-Mathieu

Francois Blanquart

Leonidas Bleris

Michael Blinov

Spencer Bliven

Jesse Bloom

Rainer Boeckmann

Joschka Boedecker

Lies Boelen

Pierre-Yves Boelle

Rafal Bogacz

Markus Böl

Peter Bond

Josh Bongard

Maciej Boni

Richard Bonneau

Roger Bonnecaze

Valerie Bonnelle

Susan Bonner-Weir

Erie Boorman

Martin Bootsma

Alla Borisyuk

Peer Bork

Alexander Borst

Giovanni Bosco

Peter Bossaerts

David Botstein

Maurizio Botta

Arezki Boudaoud

Eric Bouhassira

Anne-Laure Boulesteix

Robert Bourret

Bastien Boussau

Benjamin Bouvier

Paola Bovolenta

Grant Bowman

Gregory Bowman

Clive Bowsher

Philip Bradley

Tiago Branco

Aaron Brandes

Eduard Brandstätter

Hans Braun

A Braunstein

Johanni Brea

Michael Breakspear

Romulus Breban

Lindsay Bremner

Wieland Brendel

Eli Brenner

Michael Brenner

Naama Brenner

Steven Brenner
Paul Bressloff

Romain Brette

Ryan Brinkman

Russell Brinkworth

Abra Brisbin

James Briscoe

R. Broadhurst

Doriano Brogioli

Yana Bromberg

Ethan Bromberg-Martin

Mark Broom

Christine Brostjan

Emery Brown

Celeste Brown

Steven Brown

Kristin Brown

Sam Brown

C. Titus Brown

Chris Brown

Zachary Brown

Michael Browning

Ian Bruce

Frank Bruggeman

Søren Brunak

Nicolas Brunel

Aurelio Bruno

Jennifer Bryan

Mark Brynildsen

Gil Bub

Javier Buceta

Nicolae-Viorel Buchete

Gavin Buckingham

Christopher Buckley

Aidan Budd

David Budescu

Floris Buelens

Joerg Buescher

Lars Buesing

Michael Buhnerkempe

Alfonso Buil

Ralf Bundschuh

Anthony Burgard

Reinhard Bürger

Donald H. Burke

Anthony Burkitt

John Burnett

Jerry Busemeyer

Robin Bush

Harmen Bussemaker

Robert Butera

Daniel Butts

Markus Butz

Kevin Byrne

Kerstin Bystricky

Joana Cabral 
Amedeo Caflisch

Mehmet Caglar

Gerard Cagney

Ronald Calabrese

Vince Calhoun

Curt Callan

Laurence Calzone

Carlos Camacho

Valentina Cambiano

Colin Campbell

Jeffrey Campion

Carmen Canavier

Jianhua Cang

Robert Cannon

Xiaoyi Cao

Lulu Cao

Emidio Capriotti

Ring T. Carde

Vincent Carey

Paolo Carloni

Heather Carlson

Ross Carlson

Jens Carlsson

Anders Carlsson

Carlos Carmona-Fontaine

Maura Casadio

Rita Casadio

Donatello Castellana

Brian Castle

Jordi Catalan

Simon Cauchemez

Gillian Caughey

James Cavanagh

Rachel Cavill

Jean-Baptiste Cazier

Michele Ceccarelli

Matteo Ceccarelli

Antonio Celani

Pablo Chacon

Maurice Chacron

Monica Chagoyen

Arup Chakraborty

Farida Chamchod

Hue Chan

Nagasuma Chandra

Chandramouli Chandrasekaran

Chia-en Chang

Jeffrey Chang

Luke Chang

Dennis Chao

Claudine Chaouiya

Michael Chappell

John Chappell

Catherine Charneski

Pep Charusanti
Steve Chase

Ishanu Chattopadhyay

Eleftheria Chatzimichail

Anuradha Chauhan

Jerome Chave

Mario Chavez

Tomas E Cheatham

Gal Chechik

Rong Chen

Alan Chen

Chia-Yen Chen

Xing Chen

Wei-Hua Chen

Han Chen

Junjie Chen

Jake Chen

I-Jen Chen

Quan Chen

Liang Chen

Wen Chen

Z Chen

Chao Cheng

Cheng Cheng

Jianlin Cheng

Elissa Chesler

Lily Cheung

Sung Wook Chi

Dante Chialvo

Vikram Chib

Lauren Childs

Francis Chin

Christophe Chipot

Julien Chiquet

Haridas Chirakkal

Nakul Chitnis

Lars Chittka

Sam Cho

Samuel Cho

Adrien Chopin

Ching-Shan Chou

Tom Chou

Gerardo Chowell

Beat Christen

David Christini

Carlton Chu

Charlie Chubb

Rumi Chunara

Ho-Ryun Chung

Gennady Churakov

Luca Ciandrini

Massimo Ciccozzi

Radoslaw Cichy

Marek Cieplak

Giovanni Ciriello

Patrick Cirino 
Paul Cisek

Gerardo Cisneros

Stanca Ciupe

Jean Clairambault

Hannah Clapham

Tim Clark

Wyatt Clark

Connor Clark

Nicolas Clauvelin

Richard Clayton

Cecilia Clementi

John Clithero

Nicole Cloonan

Peter Clote

Christina Cobbold

Sarah Cobey

Simona Cocco

Luis Pedro Coelho

Ruben Coen-Cagli

Carlo Colantuoni

Claire Colas

James Cole

Michael Cole

Julio Collado-Vides

Frank Collart

Anne Collins

Giorgio Colombo

Ian Colrain

Lucy Colwell

Luca Comai

Andrew Connolly

Mark Cookson

Daniel Coombs

Jacob Cooper

Richard Cooper

Stephen Cooper

Anita Corbett

Russell Corbett-Detig

Victor Corces

Richard Cordaux

Courtney Corley

Bernat Corominas-Murtra

Manuel Corpas

Jose Correa-Basurto

Sonia Cortassa

Ivan Costa

Rui Costa

Frank Costantini

James Costello

Gary Cottrell

Zoe Cournia

Arthur Covert III

Lindsay Cowell

Susan Cox

Edward Cox
David Craig

Edmund Crampin

Rebecca Craven

Ross Cressman

Vittorio Cristini

Anton Crombach

Nicolas Crossley

Nicholas Croucher

Péter Csermely

José Cuesta

Qiang Cui

Juan Cui

Kathleen Cullen

Hermann Cuntz

Christine Currie

Christina Curtis

Carina Curto

Gennady Cymbalyuk

Tamás Czárán

Andras Czirok

Jack da Silva

Andreas Daffertshofer

Tal Dagan

Matteo Dal Peraro

Trevor Dale

Mark Daley

Yang Dan

Antoine Danchin

Thomas Dandekar

Astrid Dannenberg

Jayajit Das

Ranjan Dash

Torsten Dau

Stephen David

Maria Davidich

Diana David-Rus

Nathaniel Daw

Adriana Dawes

Troy Day

Peter Dayan

Marcus de Aguiar

Bouke de Boer

Alexandre de Brevern

Elke De Bruyne

Gianni De Fabritiis

Albert de Graaf

Wim de Grip

Bert de Groot

Willem de Haan

Monique de Jager

Jason de Koning

Gustavo de la Riva

Francisco De La Vega

Maurizio De Pittà

Jeroen de Ridder 
Jaap de Roode

Bert De Rybel

Alfonso De Simone

Ive De Smet

Marco De Vivo

Willem de Vos

Anita de Waard

Charlotte Deane

Greg DeAngelis

Dominique Debanne

Gustavo Deco

Paolo Decuzzi

Peter Dedecker

Eric Deeds

Andrea Degaetano

Victor DeGruttola

Thomas Deisboeck

Annick Dejaegere

Domitilla Del Vecchio

Matthew DeLisa

Marcel den Nijs

Vincent Denef

Sophie Deneve

Christopher Denton

Bart Deplancke

Dori Derdikman

Hartmut Derendorf

Imre Derényi

Philippe Derreumaux

Christophe Dessimoz

Nicolas Destainville

Alain Destexhe

Marie Devaine

Michael DeWeese

Colin Dewey

Diego Di Bernardo

Bendetto di Martino

Aaron Diaz

Achim Dickmanns

Casey Diekman

Paul Dijkstra

Ruxandra Dima

Dobromir Dimitrov

David Dingli

Francisco Dionisio

Dennis Discher

Oliver Distler

Markus Dittrich

Mark Ditzler

Anna Divoli

Purushottam Dixit

Narendra Dixit

Hana Dobrovolny

Andy Dobson

Andrew Doig
Brent Doiron

Nikolay Dokholyan

Socrates Dokos

Eytan Domany

Wodarz Dominik

Stefano Donadio

Jiajia Dong

Christl Donnelly

Christian Donner

I. Dorigatti

Pemra Doruker

Zsuzsanna Dosztányi

Ivan Dotu

Stéphane Douady

Daniel Dougherty

John Doyle

Finn Drablos

Jeremy Draghi

John Drake

Brian Drawert

Jacqueline Dresch

Ron Dror

Shaul Druckmann

Jan Drugowitsch

Carsten Duch

Charles Duffy

Jacques Dumais

Geneviève Dupont

Anindya Dutta

Sandrine Duverne

Rhonda Dzakpasu

Jeffrey Eaton

David Eccles

Alexander Ecker

Miguel Eckstein

Michael Economo

Evan Economo

Leah Edelstein-Keshet

Robert Edgar

Paul Edlefsen

Andrew Edwards

Matthew Edwards

Felix Effenberger

Sol Efroni

Robert Egger

Charles Eggleton

Brian Egleston

Patrick Eichenberger

Michael Eickenberg

Thorsten Eierhoff

Robyn Eijlander

Roland Eils

Miriam Eisenstein

David Elad

Sami El-Boustani 
Adrian Elcock

Wael El-Deredy

Marjet Elemans

Olivier Elemento

Mounya Elhilali

Arne Elofsson

Chris Elsik

Timothy Elston

Frank Emmert-Streib

Thierry Emonet

Heiko Enderling

Robert Endres

Jacob Engelmann

Sinead English

Bernhard Englitz

Daniel Ennis

Joshua Epstein

Harold Erickson

Bard Ermentrout

Marc Ernst

Jason Ernst

Eyal Ert

Sean Escola

Carlos Espinosa-Soto

Rino Esposito

Sylvie Estrela

Hari Eswaran

Ralf Everaers

Sebastian Eves-van den Akker

Eran Eyal

Eduardo Eyras

Elisa Fadda

James Faeder

François Fages

Adrienne Fairhall

Francesco Falciani

Martin Falcke

Daniel Falush

Hai Fang

Gang Fang

Christopher Fang-Yen

Christian Fankhauser

Hossein Farahani

Etienne Farcot

Dario Farina

Karoline Faust

Rachel Fearns

Michael Feig

Adam Feist

Jacob Feldman

Marcus Feldman

David Fell

Jérôme Feret

Neil Ferguson

Chrisantha Fernando
Evandro Ferrada

Matthew Ferrari

Dirk Fey

Ralf Ficner

Oliver Fiehn

Slawomir Filipek

Marta Filizola

James Finley

Christopher Fiorillo

Giacomo Fiorin

Daniel Fisher

Jasmin Fisher

David Fisman

Stephen Fleming

Daniel Fletcher

Alexander Fletcher

Nicolas Flores

Federico Fogolari

Brian Foley

Ariele Follis

M. Forest

Daniel Forger

Stefano Forli

Alex Fornito

Lucy Forrest

Kristoffer Forslund

Daryl Fougnie

Nicholas Foulkes

Jean-Baptiste Fournier

Douglas Fowler

Charless Fowlkes

Joanne Fox

Ingo Fründ

David Frakes

Christian Franck

Paul Francois

Marcos Frank

Steven Frank

Adam Frankish

Kevin Franks

Erik Fransen

Thomas Franz

Franca Fraternali

Tom Freeman

Jonathan Freund

Joel Freundlich

Erwin Frey

Saskia Freytag

Christian Frezza

Leonid Fridlyand

Hermann Frieboes

Caroline Friedel

Marc Friedlander

Aaron Friedman

Nir Friedman 
Tobias Friedrich

Dmitrij Frishman

Karl Friston

Robert Froemke

Simon Frost

Gang $\mathrm{Fu}$

Masami Fujiwara

Terrence Furey

Eileen Furlong

Nicholas Furnham

Robert Gütig

Terry Gaasterland

Fabrizio Gabbiani

Bruno Gaeta

Julien Gagneur

Roberto Galán

Patrick Gallagher

Jack Gallant

Jörg Galle

Alvaro Galli

Alberto Gandolfi

Sylvain Gandon

Olivier Gandrillon

Elad Ganmor

Victor Gannon

Vitaly Ganusov

Feng Gao

Fei Gao

Angel Garcia

Victor Garcia

Lana Garmire

Ruben Garrido Oter

Andrew Gartland

Paolo Gasti

Michael Gastner

M Carolyn Gates

James Gauld

Katharina Gaus

Sergey Gavrilets

$\mathrm{Hao} \mathrm{Ge}$

Nils Gehlenborg

Erol Gelenbe

Mikhail Gelfand

Guy Genin

Guy Georges

Mark Georgeson

Claude Gérard

Felipe Gerhard

Arne Gericke

Ulrich Gerland

Philip Gerlee

Samuel Gershman

Mark Gerstein

Peter Gething

Mahmoud Ghandi
Keyan Ghazi-Zahedi

Dario Ghersi

Geoffrey Ghose

Gourisankar Ghosh

Kingshuk Ghosh

Sean Gibbons

Stan Gielen

Carsten Giessing

Rona Giffard

Luis Gilarranz

Nick Gilbert

David Gilbert

Giorgio Gilestro

Jesse Gillis

Matthieu Gilson

Krzysztof Ginalski

Lisa Giocomo

Anthony Gitter

Michele Giugliano

Chad Giusti

Julijana Gjorgjieva

Stefan Glasauer

Kathryn Glass

Tilmann Glimm

Sebastian Gluth

Adam Godzik

Pranay Goel

Joshua Goldberg

Ido Golding

Byron Goldstein

Tim Gollisch

Leonardo Gollo

David Golomb

M. Gabriela Gomes

Shawn Gomez

Alexander Goncearenco

Joaquin Goni

Aitor Gonzalez

Marta Gonzalez

Didier Gonze

Benjamin Good

Kenneth Goodman

Anya Goodman

Manoj Gopalkrishnan

Uri Gophna

Raluca Gordan

Goren Gordon

Jeff Gore

Alemayehu Gorfe

Sara Gosline

Graeme Gossel

Raphael Gottardo

Mark Goulian

John Goutsias

Chris Govern 
Sidhartha Goyal

Igor Goychuk

Frauke Gräter

Michael Grabe

Manfred Grabherr

Agnieszka Grabska-Barwinska

Daniel Graham

Trevor Graham

Eleonora Grandi

Pascal Grange

Barry Grant

Michael Graupner

Patti Gravitt

Frederik Graw

Richard Gray

Jeffrey Gray

Jack Gray

Warren Grayson

C. Shawn Green

David Greenberg

Sharon Greenblum

Bryan Greenhouse

C. Greenman

Joseph Greenstein

Nicolas Gregoire

Stephan Grein

Martin Greschner

Patrick Griffin

Thomas Griffiths

Carlos Grijalva

Kalanit Grill-Spector

Ramon Grima

Andrew Grimson

Nick Grishin

Gerrit Groenhof

James Grogan

M. Michael Gromiha

Alexander Grosberg

Allison Groseth

Ivo Grosse

Moritz Grosse-Wentrup

Alan Grossfield

Christian Grove

Helmut Grubmüller

Kristina Gruden

Sonja Gruen

Dmytro Grytskyy

Jenny $\mathrm{Gu}$

Victor Guallar

Yuanfang Guan

Jeremie Guedj

Kristin Guertin

Emmanuel Guigon

William Guilford

James Gumbart
Rudiyanto Gunawan

Emre Guney

Ramneek Gupta

Todd Gureckis

Hugo Gutierrez de Teran

Michael Gutmann

Harendra Guturu

Oliver Hädicke

Jérôme Hénin

Felix Höfling

Katrin Hübner

Sauli Haataja

Suzana Hadjur

Ralf Haefner

Hiroshi Haeno

Jan Haerter

Tzachi Hagai

Daisuke Hagiwara

Stephanie Hagstrom

Matthew Hahn

Florian Hahne

Vincent Hakim

Turkan Haliloglu

Ian Hall

Timothy Hallett

Michael Hallett

Geir Halnes

Thomas Hamelryck

Daniel Hammer

Stavros Hamodrakas

Buhm Han

Andreas Handel

David Hansel

Dan Hansen

William Harcombe

Ian Harding

Marie Hardwick

Todd Hare

Simon Harris

Paul Harrison

Jennifer Harrow

HC Harsha

G Hart

Gal Haspel

Soha Hassoun

Alan Hastings

Stefan Haufe

James Havranek

Michael Hawrylycz

Satoru Hayasaka

Shigehiko Hayashi

Eric Hayden

John-Dylan Haynes

Steven Hayward

Vincent Hayward 


\section{PLOS Compuratronal}

William Hazelton

Guillaume Hollard

DaiHai He

David Holloway

David Heckmann

Petter Holme

Tyson Hedrick

Dieter Heermann

Susan Holmes

lan Holmes

Ines Heiland

Christine Heitsch

Jeffrey Holmes

Richard Heitz

Hermann-Georg Holzhütter

Brian Helmke

Christian Hong

Barry Honig

Volkhard Helms

Guillaume Hennequin

Stefan Hoops

Thomas Hopf

Matthias Hennig

Timothy Horiuchi

Bruce Henry

Christopher Henry

Niel Hens

Burkhard Hense

Scott Hensley

Michael Henson

Jean-Karim Heriche

Rigoberto Hernandez

Esteban Hernandez Vargas

Miguel Herrero

Markus Herrgard

Andreas Herrmann

Jeremy Horst

Tingjun Hou

Conor Houghton

Thomas House

Eugene Houseman

Elizabeth Housworth

Martin Howard

Jonathon Howard

Clare Howarth

Brendan Howlin

Li Hsu

Jialu $\mathrm{Hu}$

Xiao $\mathrm{Hu}$

Eva Herrmann

Pascal Hersen

Zhenjun $\mathrm{Hu}$

Uri Hershberg

Gangqing $\mathrm{Hu}$

Hanspeter Herzel

Xuhui Huang

Peter Heutink

Stephen Hewitt

Matthew Hibbs

Kevin Hicks

Christian Hilbe

Mark Hildebrand

Niu Huang

Jeffrey Huang

Kerwyn Huang

Jing Huang

Jochen Hub

Greg Huber

Sean Hill

Heinrich Huber

Alison Hill

Matthew Hudson

Grant Hill-Cawthorne

Corey Hudson

Ronald Hills

Jane Hillston

Ramon Huerta

Sean Humbert

Konrad Hinsen

Gerhard Hummer

Tomoo Hirano

Mark Humphries

Lynette Hirschman

Martin Hjortsø

William Hlavacek

Vanthai Hoang

Birte Höcker

Toby Hocking

Ina Hoeschele

Michael Hoffman

C. Antony Hunt

Daniel Huson

Cendri Hutcherson

Alexander Huth

Gyorgy Hutvagner

Quentin Huys

Eun Jung Hwang

Fahmeed Hyder

Daniel Hoffmann

Changbong Hyeon

Sabrina Hoffmann

Timon Idema

Kay Hofman

Ales Iglic

John Hogenesch

Oleg Igoshin

Arun Holden

Nevena llieva-Litova

Benjamin Holder

Chris Illingworth 
Princess Imoukhuede

Kiao Inthavong

Ed Ionides

Herve Isambert

Rezarta Islamaj Dogan

Yuval Itan

Viktor Ivanov

Junji Iwahara

Masoumeh Izadi

Dean Jackson

Robert Jacobs

Donald Jacobs

Anders Jacobsen

Matthew Jacobson

Johannes Jaeger

Frank Jaekel

Ajay Jain

Kavita Jain

Ciriyam Jayaprakash

Rick Jenison

Garrett Jenkinson

Lars Jensen

Oliver Jensen

Jan Jensen

Paul Jensen

Livnat Jerby Arnon

Robert Jernigan

Chris Jewell

Aaron Jex

Hongkai Ji

Shuiwang Ji

Wei Ji Ma

Yi Jiang

Hui Jiang

Bing-Hua Jiang

Yang Jiao

Alexandra Jilkine

Guangxu Jin

Dezhe Jin

Viktor Jirsa

Peter Johansen

Philip Johnson

Thomas Johnson

Henry Johnston

Wilsaan Joiner

Vladimir Jojic

Thibaut Jombart

Inge Jonassen

James Jones

Siddhartha Jonnalagadda

Rebecka Jornsten

Sanjay Joshi

Kresimir Josic

Daniel Jost

Peter Jung
Irwin Jungreis

Mordechai Juni

Ivan Junier

Lukas Käll

Jaap Kaandorp

Joe Kable

Lars Kaderali

Marcus Kaiser

Kostas Kalogeropoulos

Christel Kamp

Bjoern Kampa

Takahisa Kanekiyo

Shuli Kang

Ingmar Kanitscheider

Jitendra Kanodia

Eva Kanso

Rowland Kao

John Karanicolas

Jan Karbowski

George Karniadakis

Mikko Karttunen

Matthias Kaschube

Eugene Kashdan

Robert Kass

Peter Kasson

William Kath

Saul Kato

Michael Katze

Laura Kaufman

Kamran Kaveh

Kendrick Kay

Mitsunori Kayano

Hilal Kazan

Yiannis Kaznessis

Sunduz Keles

Douglas Kellogg

Daniel Kelly

J.A. Scott Kelso

Christopher Kempes

Eben Kenah

Terry Kenakin

Thomas Kepler

Andrew Kern

Rex Kerr

Can Kesmir

Sarah Kessans

Sinan Keten

Anmar Khadra

Ahmad Khalil

Fatemeh Khalili-Araghi

Mustafa Khammash

Himanshu Khandelia

Raya Khanin

Bhavin Khatri

George Khelashvili 
Reza Khodarahmi

Roozbeh Kiani

Akinori Kidera

Andrzej Kierzek

Volker Kiessling

Daisuke Kihara

David Kikuchi

Zachary Kilpatrick

Yoo-Ah Kim

Jae Kyoung Kim

Philip Kim

Junhyong Kim

Taeyoon Kim

Yongsoo Kim

Sanguk Kim

Seyoung Kim

Marek Kimmel

Oliver King

Aaron King

Carl Kingsford

Tamara Kinzer-Ursem

Mark Kirkpatrick

Denise Kirschner

Istvan Kiss

Steffen Klamt

Per Johan Klasse

Jeffery Klauda

Max Kleiman-Weiner

Teri Klein

Ulrich Kleinekathoefer

Jurgen Kleine-Vehn

David Kleinfeld

Judith Klein-Seetharaman

Steven Kleinstein

Dmitri Klimov

David Klinke

Don Klinkenberg

Edda Klipp

Peter Kloeden

Yuval Kluger

Stefan Klumpp

Jonas Knape

David Knapp

Jefferson Knight

Dan Knights

David Knill

Andreas Knoblauch

Jaroslav Koča

Dmitry Kobak

Ina Koch

Peter Kochunov

Katia Koelle

Heinz Koeppl

Oliver Kohlbacher

Markus Kollmann
Rachel Kolodny

Anatoly Kolomeisky

Natalia Komarova

Michal Komorowski

Genevieve Konopka

Anna Konstor

Bette Korber

Tamás Korcsmáros

Alon Korngreen

Kirill Korolev

Sergei Kosakovsky Pond

Katya Kosheleva

Mickey Kosloff

Andrej Kosmrlj

Michael Kosorok

George Kostopoulos

Alexei Koulakov

Markos Koutmos

Roger Kouyos

Gregor Kovacic

Mehmet Koyuturk

Dima Kozakov

James Kozloski

Ian Krajbich

Mark Kramer

Bernhard Kramer

Natalio Krasnogor

Andreas Kremling

Robert Kretsinger

Elmar Krieger

Arjun Krishnan

Vessela Kristensen

Joachim Krug

Karsten Kruse

Sergey Kryazhimskiy

John Kubie

Roman Kuc

Andrzej Kudlicki

Arvind Kumar

Anshul Kundaje

Ambarish Kunwar

Chih-Horng Kuo

Daisuke Kurabayashi

Lukasz Kurgan

Shinya Kuroda

Edo Kussell

Rafal Kustra

Jason Kutch

Vladimir Kuznetsov

Taejoon Kwon

Inchan Kwon

David La

Giancarlo La Camera

Vincent Lacroix

Alain Laederach 
Fereshteh Lagzi

Nicola Lai

Sirish Lakkaraju

Renaud Lambiotte

Jan Lammerding

Frank Lammert

Meytal Landau

Dirk Landgraf

Gary Landreth

Christian Landry

Samuel Landry

Michael Landy

Jenna Lang

Christopher Langmead

Daniel Larson

Michael Lassig

Peter Latham

Kenneth Latimer

Vito Latora

Andy Lau

Ken Lau

Reinhard C. Laubenbacher

Douglas Lauffenburger

Tim Laumann

Gilles Laurent

Michel Laurin

Christophe Lavelle

Richard Lavery

Jeffrey Lawrence

Michael Lawrence

Christopher Lawrence

Daniel Laydon

Themis Lazaridis

Matthew Lazzara

Nicolas Le Novère

Arthur Leblois

Paola Lecca

Gwenael Leday

Shane Lee

Dong-Yup Lee

Michael Lee

Jaejin Lee

Hyunju Lee

Sangwan Lee

Insuk Lee

Kyongbum Lee

Stefan Legewie

Joseph Lehar

Laurent Lehmann

Ming Lei

Hong Lei

Christian Leibold

Bertrand Lemasson

Louis Lemieux

Marc Lenburg
Máté Lengyel

Martin Lercher

Nicholas Lesica

Henry Leung

Erez Levanon

Gabriel Leventhal

Anna Levina

Michael Levitt

Sivan Leviyang

Emmanuel Levy

Yaakov Levy

Daniel Lew

Nathan Lewis

Bryan Lewis

Paul Lewis

Fran Lewitter

Klaus Ley

Lei Li

Hongzhe Li

Cong $\mathrm{Li}$

Tong $\mathrm{Li}$

Chunhe Li

Pan Li

Wei Li

Peng Li

Yue Li

Wei Li

Wenyuan Li

Jingjing $\mathrm{Li}$

$\mathrm{Li} \mathrm{Li}$

Nan Li

Xiaoyu Liang

Han Liang

Jie Liang

Ran Libeskind-Hadas

Jonathan Licht

Oliver Lichtarge

Bomyi Lim

Jiaojiao Lin

Kevin Lin

Martin Linden

Stinus Lindgreen

Kresten Lindorff-Larsen

Michal Linial

Sébastien Lion

Tomasz Lipniacki

Veronique Lisi

Ashok Litwin-Kumar

Yunlong Liu

Tao Liu

Robert Liu

Song Liu

Dennis Livesay

Joseph Lizier

James Locke 
Gerald Loeb

Benjamin Logsdon

Kirk Lohmueller

Nir London

Mickey London

André Longtin

Lit-Hsin Loo

Liliana Losada

Yoram Louzoun

Michael Love

Simon Lovell

David Lovell

Claude Loverdo

Pedro Lowenstein

Long Lu

Benzhuo Lu

James Lu

Xinghua Lu

David Lubensky

Sharon Lubkin

Joseph Lucas

Fabio Luciani

David Lukatsky

Desmond Lun

Daniel Lundin

Gerton Lunter

Jingqin Luo

Zewei Luo

Andrei Lupas

Javier Luque

G.W. Gant Luxton

Jeremy Lynch

Shina Caroline Lynn Kamerlin

Grant Lythe

Katrina Lythgoe

Alexander Lyubartsev

Lan Ma

Viktor Müller

Feilim Mac Gabhann

David MacAlpine

Ben MacArthur

Christopher MacDermaid

Malcolm Maclver

Jakob Macke

Alexander MacKerell

Paul Macklin

Jason MacLean

Shev MacNamara

Jeffry Madura

Carsten Magnus

Radhakrishnan Mahadevan

L. Mahadevan

Narendra Maheshri

Tarek Mahfouz

Volker Mai
Thomas Mailund

Francois Major

Alpeshkumar Malde

Sairam Mallajosyula

Thérèse Malliavin

Prema Latha Mallipeddi

Brian Malone

Pascal Mamassian

Piero Manfredi

Matthias Mann

Ranjan Mannige

Lisa Manning

Poramate Manoonpong

Manu

Luca Maragliano

Costas Maranas

Daniel Marbach

Adam Marblestone

Paolo Marcatili

Edward Marcotte

Eve Marder

Adil Mardinoglu

Andrea Mari

Daniele Marinazzo

John Marioni

Paul Marjoram

Alan E Mark

Doug Markant

Scott Markel

Florian Markowetz

Phineus Markwick

Siewert Jan Marrink

Joseph Marsh

Javier Martin

Alfonso Martinez-Arias

Marc Marti-Renom

Yosef Maruvka

Dominik Marx

Manja Marz

Laura Masgrau

Sergei Maslov

Christopher Mason

Timothée Masquelier

Marcello Massimini

Jean-Baptiste Masson

Francesco Massucci

Naoki Masuda

William Mather

Tom Matheson

Alexander Mathis

Frederick Matsen

Noriyuki Matsuda

Maurizio Mattia

Vincent Mauro

Eric May 
Alberto Mazzoni

Mark McAuley

James McCaw

Megan McClean

Josh McDermott

Daniel Mcdonald

James McFarland

Joel McGlothlin

Alison McGuigan

Joseph McGuire

Alice McHardy

David Mclver

William McLaughlin

Robert McMeeking

David McMillen

James McNally

Duccio Medini

Jan Medlock

Michael Mehan

Ahmed Mehdi

Ramit Mehr

Feryal Mehraban Pour Behbahani

Deavn Mehrotra

Pankaj Mehta

Martin Meier-Schellersheim

Hans Meinhardt

Manuel Melo

Francisco Melo

Raoul-Martin Memmesheimer

Pedro Mendes

Yilin Meng

Filippo Menolascina

Vinod Menon

Hugo Merchant

Dan Mercola

Vicky Merhej

Roeland Merks

Stefano Merler

Samy Meroueh

Martha Merrow

Arnaud Messé

C. Jessica Metcalfe

Dirk Metzler

Ralf Metzler

Markus Meuwly

Austin Meyer

Michelle Meyer

Michael Meyer-Hermann

Ethan Meyers

Rafael Meza

Hongyu Miao

George Mias

Anushka Michailova

Magali Michaut

Cristian Micheletti
Tom Michoel

Daniel Mietchen

David Miguez

Alexey Mikaberidze

Hiroaki Miki

Tijana Milenkovic

Olgica Milenkovic

Joel Miller

Yifat Miller

Lee Miller

Kai Miller

John Milton

Leonid Mirny

Yuriy Mishchenko

Natasa Miskov-Zivanov

John Mitchell

Colleen Mitchell

Julie Mitchell

Sorin Mitran

Satoru Miyano

Osamu Miyashita

Jeff Moehlis

Mohammad Mofrad

Alex Mogilner

James Moir

Jaap Molenaar

Sven Moller-Tank

Remi Monasson

Gianluigi Mongillo

Nick Monk

Ernest Montbrio

Enrique Monte

Stephen Montgomery

Luca Monticelli

M. Moody

Paul Moore

Thierry Mora

Rosalyn Moran

Massimo Morbidelli

Ruben Moreno-Bote

Kari Morgan

Martin Morgan

Dimitrios Morikis

Alexandre Morozov

Richard Morris

Quaid Morris

Richard Morton

Olaf Mosbach-Schulz

Roberto Mosca

Alan Moses

Mathieu Moslonka-Lefebvre

Sara Mostafavi

Rafal Mostowy

Fumio Motegi

Ahmed Moustafa 
Andrés Moya

Aurelio Moya Garcia

Kianoush Nazarpour

Florian Mueller

Christian Mueller

Stephen Muench

Andrew Mugler

Sayan Mukherjee

Subhadeep Mukhopadhyay

Nicola Mulder

Sarah Muldoon

Adrian Mulholland

Christopher Mundt

Brian Munsky

Ivan Mura

Cyrill Muratov

Robert Murphy

Chris Murray

Philip Murray

Richard Murray

John Murray

Ben Murrell

Arvind Murugan

Ferdinando Mussa-Ivaldi

Ville Mustonen

Vivek Mutalik

Chris Myers

Chad Myers

Christopher Myers

Jean-Pierre Nadal

Zoltan Nadasdy

Elena Nadezhdina

Farzan Nadim

Suhita Nadkarni

Robert Nadon

Niranjan Nagarajan

Valentin Nagerl

Deepak Nagrath

John Nagy

Mate Nagy

Rafael Najmanovich

Suguru Nakagawa

Luay Nakhleh

Aurélien Naldi

Jin-Wu Nam

Kwangho Nam

Gautham Namasivayam

Atsushi Nambu

Olivier Namy

Giri Narasimhan

Rishikesh Narayanan

Jatin Narula

Thomas Naselaris

Matthew Nassar

Matt Nassar

Francisco Navarro

Peter Neal

Chris Neale

Emre Neftci

Richard Neher

Ali Neishabouri

Israel Nelken

Celeste Nelson

Peter Nelson

Ilya Nemenman

Goran Nenadic

Irina Nesmelova

Theoden Netoff

Thomas Nevian

Jay Newby

Stuart Newman

Fengyun $\mathrm{Ni}$

Colin Nichols

Jeffrey Nickerson

Dan Nicolae

Stamatios Nicolis

Qing Nie

Steven Niederer

Jens Nielsen

Morten Nielsen

Evgeni Nikolaev

Roland Nilsson

Lennart Nilsson

Alex Ninfa

Hafumi Nishi

Chuanxin Niu

Masha Niv

Yael Niv

Frank Noé

Taishin Nomura

Rachel Norman

Sergei Noskov

Kirill Nourski

Houtan Noushmehr

Régis Nouvian

Daichi Nozaki

Sergey Nuzhdin

Mike Oaksford

Anna Ochab-Marcinek

Alejandro Ochoa

Lauren O'Connell

Gisele Oda

John O'Doherty

Lauren O'Donnell

Amy Odum

Thomas Oertner

Peter Oeschlager

Robert D Ogilvie

Uwe Ohler

Marie Öhman 


\begin{tabular}{|c|c|}
\hline & $\begin{array}{l}\text { Balázs Papp } \\
\text { Francesco Pappalardo }\end{array}$ \\
\hline Masato Okada & Leonardo Pardo \\
\hline Eckehard Olbrich & Néstor Parga \\
\hline Timothy O'Leary & Laxmi Parida \\
\hline Aude Oliva & Memming Park \\
\hline Bruno Olshausen & Juyong Park \\
\hline Jose Onuchic & Christopher Park \\
\hline Thom Oostendorp & Leopold Parts \\
\hline Hans Op de Beeck & Mercedes Pascual \\
\hline Lulla Opatowski & Annalisa Pastore \\
\hline Lance Optican & Kiran Patil \\
\hline Jean-Jacques Orban de Xivry & Kaustubh Patil \\
\hline Jill O'Reilly & Etienne Patin \\
\hline Paul O'Reilly & Julie Pavlin \\
\hline Christine Orengo & Gordana Pavlovic-Lazetic \\
\hline Patricio Orio & Klaus Pawelzik \\
\hline Amy Orsborn & Florencio Pazos \\
\hline Pedro Ortega & Jean Peccoud \\
\hline Remus Osan & Morten Pedersen \\
\hline James Osborne & Dana Pe'er \\
\hline Anne Osbourn & Shayn Peirce \\
\hline Anne-Marie Oswald & Catherine Pellat-Deceunynck \\
\hline Hans Othmer & Joelle Pelletier \\
\hline Sarah Otto & Jian Peng \\
\hline Francis Ouellette & William Penny \\
\hline Thomas Ouldridge & John Pepper \\
\hline Zhengqing Ouyang & Matjaz Perc \\
\hline Markus Owen & Jose Pereira-Leal \\
\hline Dylan Owen & Alan Perelson \\
\hline Diego Oyarzún & Alberto Perez \\
\hline Ertugrul Ozbudak & Alex Perkins \\
\hline Banu Ozkan & Sarah Perkins \\
\hline Elif Ozkirimli Olmez & Andrea Perna \\
\hline Alberto Paccanaro & Remo Perozzo \\
\hline Roland Pache & Robert Peterka \\
\hline Jorge Pacheco & Megan Peters \\
\hline Emanuele Paci & Rasmus Petersen \\
\hline Christopher Pack & Carsten Peterson \\
\hline Andrea Pagnani & Karin Petrini \\
\hline Debnath Pal & Giovanni Pezzulo \\
\hline Sean Palecek & Jim Pfaendtner \\
\hline Günther Palm & Michael Pfeiffer \\
\hline Lucy Palmer & Benjamin Pfeuty \\
\hline Stephanie Palmer & Jean-Pascal Pfister \\
\hline Arthur Palmer & Andrew Philippides \\
\hline Stefano Palminteri & Francesco Piazza \\
\hline Anna Panchenko & Simone Pigolotti \\
\hline Liam Paninski & Jonathan Pillow \\
\hline Jasmina Panovska-Griffiths & Sergei Pilyugin \\
\hline Tanate Panrat & Flávio Pinheiro \\
\hline Stefano Panzeri & Dimitris Pinotsis \\
\hline Daniela Paolotti & Paulo Pinto \\
\hline Panagiotis Papasaikas & Davide Piovesan \\
\hline Jason Papin & Andrei Pisliakov \\
\hline
\end{tabular}


Xaq Pitkow

Francisco Planes

Mike Plank

Dietmar Plenz

Sylvia Plevritis

Maksim Plikus

Joshua Plotkin

Michael Poidinger

Panayiota Poirazi

Daniel Polani

Rafael Polania

Piero Poletti

Philip Polgreen

Régis Pomès

Adrian Ponce-Alvarez

Mikhail Ponomarenko

Beatriz Pontes

Art Poon

Tatiana Popova

Maurizio Porfiri

Francesc Posas

Juan Poyatos

Christian Pozzorini

Nataša Pržulj

Robert Preissner

Drazen Prelec

Tony Prescott

Steven Prescott

Steve Presse

Nathan Price

Astrid Prinz

U. Deva Priyakumar

William Probert

Sotiris Prokopiou

Vasilis Promponas

Nicholas Provart

Davide Provasi

Astero Provata

Paolo Provero

Przemyslaw Prusinkiewicz

Teresa Przytycka

Jingzhi Pu

Jose Puglisi

Alfredo Pulvirenti

Marco Punta

Jeremy Purvis

Feng Qi

Zhen Qi

Yuan Qi

Wenfeng Qian

Jiang Qian

Hong Qian

Yan Qin

Zhaohui Qin

Zhilin Qu
Changqin Quan

Gunnar Rätsch

Mikhail Rabinovich

Joshua Rabinowitz

Julien Racle

Todd Rae

Gregor Rainer

Arjun Raj

Sohini Ramachandran

Ravishankar Ramachandran

Kumaran Ramamurthi

E. Prabhu Raman

Stephen Ramsey

Padmini Rangamani

Rajiv Ranganathan

Christopher Rao

Nalam Rao

Benjamin Raphael

Wouter-Jan Rappel

Michael Rappolt

Maria Anna Rapsomaniki

Matthias Rarey

Garvesh Raskutti

David Rasmussen

Muruhan Rathinam

Oliver Ratmann

Thomas Rattei

Magnus Rattray

Erzsebet Ravasz Regan

Barak Raveh

J. Christian Ray

Soumya Raychaudhuri

Simon Rayner

Montague Read

Cyril Reboul

Pedro Reche

Mario Recker

Michael C. Reed

Jennifer Reed

Roland Regoes

Alexandra Reichenbach

Mark Reimers

Pieter Rein ten Wolde

Robert Reiner

Cynthia Reinhart-King

Johannes Reisert

Katarzyna Rejniak

Michiel Remme

Michael Rendl

Olivier Restif

Nathalie Reuter

Eduard Reznik

Seung Rhee

Nick Rhind

Ruy Ribeiro 
Federico Ricci Tersenghi

Magnus Richardson

Stephen Richmond

Nicole Riddle

Andrea Riebler

Maximilian Riesenhuber

Guillem Rigaill

Michel Rigoulet

Steven Riley

Claire Rind

John Rinzel

Karsten Rippe

Herre Jelger Risselada

Marylyn Ritchie

Petra Ritter

Marcelo Rivas-Astroza

Vincent Robert

James Roberts

David Robertson

Richard Robins

Peter Robinson

Simon Robson

Walter Rocchia

Eduardo Rocha

Luis Rocha

Blanca Rodriguez

Isabel Rodriguez Barraquer

Pieter Roelfsema

Henk Roelink

Jose Rojas

Sandro Romani

Lilia Romdhane

Ethan Romero-Severson

Yannick Rondelez

Libin Rong

Angelo Rosa

Gabriel Rosser

Saharon Rosset

Simona Rossi

Igor Rouzine

Alex Roxin

Sushmita Roy

Christopher Rozell

Pau Rué

Leonid Rubchinsky

Dan Rubenstein

Joshua Rubin

Jonathan Rubin

Mikail Rubinov

Amir Rubinstein

Michael Rudd

Claus Rueffler

Matthew Ruffalo

Sandra Rugonyi

Peter Ruoff
Lasse Ruokolainen

Eytan Ruppin

Robert Russell

Michael Rust

Gustaf Rydell

Andrey Rzhetsky

Laura Sacerdote

Frank Sachse

Julio Saez-Rodriguez

Dov Sagi

Maneesh Sahani

Debashis Sahoo

Surinder S. Sahota

Naruya Saitou

Lisa Saksida

Kourosh Salehi-Ashtiani

Howard Salis

Alexandre Salvador

Julia Salzman

C. Daniel Salzman

Ines Samengo

Ilan Samish

Maria Samsonova

Karissa Sanbonmatsu

Roberto Sanchez

Conner Sandefur

Guido Sanguinetti

Michel Sanner

Vijayalakshmi Santhakumar

Francisco Santos

Mauro Santos

Vinay Satish Kumar

Uwe Sauer

Herbert Sauro

Olivier Saut

Thomas Sauter

Jeff Saven

Christina Savin

Nathaniel Sawtell

Akansha Saxena

Samuel Scarpino

Lars Schäfer

Jörg Schaber

William Schafer

Jeffrey Schall

Christoph Schaniel

Konrad Scheffler

Michael Schellenberger Costa

Birgit Schiøtt

Steven Schiff

Joshua Schiffer

Marcel Schilling

Mark Schira

Alexander Schliep

Patrick Schloss 
Christoph Schmal

Sebastian Schmeier

Michael Schmuker

Ralf Schneggenburger

Birgit Schoeberl

Alexander Schoenhuth

Paul Schrater

Susanne Schreiber

Friederike Schueuer

Marcel Schulz

David Schulz

Peter Schuster

David Schwab

James Schwaber

David Schwartz

Daniel Schwartz

Russell Schwartz

Pamela Schwartzberg

Roland Schwarz

Nicolas Schweighofer

Michael Schwemmer

Joost Schymkowitz

Marco Scianna

Michelle Scott

Jacob Scott

David Searls

Timothy Secomb

Daniel Seeliger

Gunnar Seemann

Emre Sefer

Rebecca Segal

Nicola Segata

Ayellet Segrè

Terrence Sejnowski

Inbal Sela

Luc Selen

Guy Sella

Shamik Sen

Hanoch Senderowitz

Irene Sendina-Nadal

Alessandro Senes

Patrick Senet

Ryan Senger

Anirvan Sengupta

Walter Senn

Cathal Seoighe

Chaok Seok

James Sethna

Alessandro Sette

Manu Setty

Nikolaos Sgourakis

Shawn Shadden

Joshua Shaevitz

Patrick Shafto

Nigam Shah
Sohrab Shah

Premal Shah

Eugene Shakhnovich

Maoz Shamir

Shihab Shamma

Yibing Shan

Maryam Shanechi

Catherine Shang

Daryl Shanley

Tali Sharot

Gemma Sharp

Tatyana Sharpee

Thomas Sharpton

Jared Shaw

Eric Shea-Brown

Mark Shein

Jana Shen

Xiling Shen

Yanhong Shi

Xinghua Shi

Lei Shi

Darryl Shibata

Tetsuo Shibuya

Hideaki Shimazaki

Tom Shimizu

Yishai Shimoni

Jay Shin

Wataru Shinoda

Hiroki Shirai

Abbas Shirinifard

George Shirreff

G.V. Shivashankar

Eli Shlizerman

Mark Shlomchik

Harel Shouval

Jianwei Shuai

Igor Shuryak

Heike Siebert

Eric Siggia

Wendy Silk

Jerson Silva

Pamela Silver

Patrick Simen

Peter Simmons

Scott Simon

Eero Simoncelli

Daniel Simons

Thomas Simonson

Julie Simpson

Suzanne Sindi

Abhyudai Singh

Karan Singh

Sheila Singh

Saurabh Sinha

Photini Sinnis 
Fabian Sinz

Jacobo Sitt

Per Sebastian Skardal

Maja Skataric

Peter Skelsey

Frances Skinner

Brian Skinner

Jan Skotheim

Brian Skyrms

Jamie Sleigh

Boris Slepchenko

Donna Slonim

Timo Smieszek

Roger Smith

Eric P. Smith

Maurice Smith

Tim Smith

V. Anne Smith

Amber Smith

Marcus Smolka

Michael Smotherman

Gordon Smyth

Erik Snapp

Michael Sneddon

Berend Snel

Kim Sneppen

James Sneyd

Jacky Snoep

Claudio Soares

Eric Sobie

Joshua Socolar

Johannes Soeding

Juan Soler

Soroosh Solhjoo

Trygve Solstad

George Somero

David Somers

Charlotte Soneson

Jimin Song

Sen Song

Nikolaus Sonnenschein

Andrew Sornborger

Cinque Soto

Christoph Sotriffer

Andrea Sottoriva

Andrew Spakowitz

Rainer Spang

Lee Spector

Michael Spivey

Jiri Sponer

Glen Spraggon

David Sprinzak

Sudharsan Sridharan

Jagan Srinivasan

Mandyam Srinivasan
Ganesh Sriram

Ranjan Srivastava

Peter Stadler

Dominic Standage

Kenneth Stanley

Anna Stary-Weinzinger

Oliver Stegle

Gary Stein

Dov Stekel

Federico Stella

Jorg Stelling

Ulrich Stelzl

Martin Stemmler

Klaas Stephan

Dagmar Sternad

Ralf Steuer

Alasdair Steven

Ian Stevenson

Klaus Stiefel

Gautier Stoll

Lewi Stone

Barbara Stranger

Birgit Strodel

Roland Strong

Michael Stumpf

Andrew Su

Shankar Subramaniam

Gurol Suel

Piotr Suffczynski

Sergei Sukharev

Christopher Summerfield

David Sumpter

Sean Sun

Margaret Sunde

Vikram Sunkara

Susan Sunkin

Richard Superfine

Granger Sutton

Samir Suweis

Maciej Swat

Gyorgy Szabo

Ewa Szczurek

Ágnes Tóth-Petróczy

Joel Tabak

Shoji Takada

Daniel Takahashi

Nobuto Takeuchi

Kai Tan

Ming Tan

Mark Tanaka

Sorin Tanase Nicola

Sishi Tang

Chao Tang

Yinjie Tang

Haixu Tang 
Bertrand Tanner

Mounir Tarek

Peter Tass

Ozlem Tastan Bishop

Nicholas Tatonetti

Diethard Tautz

Iman Tavassoly

Merryn Tawhai

Jordan Taylor

Ronald Taylor

William Taylor

Christopher Taylor

Tracy Teal

James Tee

Tobias Teichert

Vladimir Teif

Marcus Teixeira

Aurelien Tellier

David Terman

Glenn Tesler

Bas Teusink

Shivendra Tewari

Johannes Textor

Mukund Thattai

Lucas Theis

Fabian Theis

Evangelos Theodorou

Frédéric Theunissen

Ines Thiele

Jean-Philippe Thivierge

Peter Thomas

Paul Thomas

Dennis Thomas

Morgane Thomas-Chollier

Wesley Thompson

Kurt Thoroughman

Simon Thorpe

Ian Thorpe

Xiao-Jun Tian

Chaoguang Tian

Alessandro Ticchi

D. Peter Tieleman

Axel Tiessen

Cezar Tigaret

Michael Tildesley

Marc Timme

Leleu Timothee

Brian Tjaden

Robert Tjian

Gašper Tkačik

Kai Toellner

Andreas Tolias

Dean Tolla

Iuliana Toma-Dasu

Giovanna Tomaiuolo
Cristian Tomasetti

Dan Tompkins

Tina Toni

Giulio Tononi

Maya Topf

Stefano Toppo

Bruce Torbett

Adriano Tort

Yoshihiro Toya

Taro Toyoizumi

Zlatko Trajanoski

Mark Transtrum

Cole Trapnell

Michael Tress

Jochen Triesch

Edward Trifonov

Antoine Triller

Wilson Truccolo

Anya Tsalenko

Krasimira Tsaneva-Atanasova

Doris Tsao

Lev Tsimring

Misha Tsodyks

Zhidong Tu

Elio Tuci

Lisa Tucker-Kellogg

Greg Tucker-Kellogg

Mark Tuckerman

Tamir Tuller

Nurcan Tuncbag

Paul Tupper

Srini Turaga

John Turchi

Adrian Turjanski

Joel Turner

Brandon Turner

Ten Tusscher

Jack Tuszynski

John Tyson

Balazs Ujfalussy

Nachum Ulanovsky

Igor Ulitsky

Jakob Ulmschneider

Myco Umemura

David Umulis

Peter Man-Un Ung

Marius Usher

David Ussery

Nagarajan Vaidehi

Sandor Vajda

Ady Vaknin

Ilya Vakser

Faramarz Valafar

Edelmira Valero Ruiz

Francisco Valero-Cuevas 
Taufik Valiante

Robert van Beers

Willem van Berkel

Petra Van Damme

Martijn van de Bunt

Hugo Van den Berg

Martijn van den Heuvel

David van der Spoel

Arjan van der Vaart

Stijn van Dongen

Fred van Eeuwijk

Celia Van Gelder

Leo van lersel

Erik van Nimwegen

Arjen van Ooyen

Jaap van Pelt

Natal van Riel

Mark van Rossum

Benjamin VanderSluis

Fabio Vandin

Rufin VanRullen

Jeffrey Varner

Charles Vaske

Stefano Vassanelli

Ilpo Vattulainen

Timothy Vaughan

Dimitrios Vavylonis

Catia Vaz

Gianluigi Veglia

Michele Vendruscolo

Paul Verschure

Jean-Philippe Vert

Cordula Vesper

Allegra Via

Cecile Viboud

Raul Vicente

Jonathan D. Victor

Brani Vidakovic

Marc Vidal

Edward Vigmond

Jose Vilar

Bjarni Vilhjalmsson

Arunachalam Vinayagam

Martin Vinck

Yngvild Vindenes

Alessia Visconti

Saraswathi Vishveshwara

Ivo Vlaev

Yoram Vodovotz

Vincent Voelz

Christopher Voigt

Raphael Voituriez

Erik Volz

Tobias von der Haar

Christof Von der Malsburg
Max von Kleist

Gregory Voth

Bradley Voytek

Edward Vul

Emilia Vynnycky

Thomas Wachtler

Rebecca Wade

Alex Wade

Eric-Jan Wagenmakers

Dylan Wagner

Aleksandra Walczak

Jerome Waldispühl

Patrick Walker

Michael Wall

Jeffrey Wall

Chris Wallace

Jacco Wallinga

Dirk Walther

Gilles Wandeler

Brian Wandell

Xiao Wang

Junwen Wang

DeLiang Wang

Jing Wang

Xiaowo Wang

Tao Wang

Ting Wang

Hongyun Wang

Zhihui Wang

Peter Wang

Qi Wang

Kai Wang

Xiao-Jing Wang

Bo Wang

Jin Wang

Xujing Wang

Rui Wang

John Wann

Melissa Ward

Joanna Wares

Andrew Wargo

Tandy Warnow

Tsjerk Wassenaar

Helen Wearing

Robert Weatheritt

Glenn Webb

Barbara Webb

Caleb Webber

Gerald Weber

Michael Webster

Matti Weckström

Wolfram Weckwerth

Michael Wehr

Gilbert Weidinger

Martin Weigt 
Aaron Weimann

Geraldine Wright

Ariel Weinberger

Yu-Wei Wu

Patrick Weinkam

Yinghao $\mathrm{Wu}$

Harel Weinstein

Zhijin Wu

Matthew Weirauch

Joseph Wu

Dahlia Weiss

Joshua Welch

Edward Wenger

Uno Wennergren

Alex Chi Wu

Wei Wu

Xuebing Wu

Haishan Wu

Wolfgang Wenzel

Dongying Wu

Benjamin Werner

Stefan Wuchty

Joel Wertheim

Jevin West

Christoph Wuelfing

Pål Westermark

Joao Xavier

Robert Wheeler

Richard Wheeler

Yu Xia

Yi Xiao

Bei Xiao

Nicole Wheeler

Chris Whidden

Laura White

John White

Paul Whitford

Steven Whitten

David Whitworth

Xiao Xiao

Lu Xie

Xiaohui Xie

L Xie

Jinchuan Xing

Ren Xu

Wenfang $\mathrm{Xu}$

Michael Wibral

Jinbo $X u$

$\mathrm{Li} \mathrm{Xu}$

Andreas Wieland

Larry Yaeger

Sadie Wignall

Herman Wijnen

Steven Wiley

Brian Wilhelm

Claus Wilke

Loren Williams

Ross Williamson

Robert Wilson

Bridget Wilson

Eitan Yaffe

Nir Yakoby

Yoshihiro Yamanishi

Brian Yandell

Wei Yang

Xingzhou Yang

Wan Yang

Ence Yang

Yang Yang

Tal Yarkoni

Michael Yarus

Jonathan Winawer

Andrew Yates

Erik Winfree

Ned Wingreen

Rudolf Winklbauer

Jian Ye

Fei Ye

Roland Winkler

Peter Winn

Patrick Wintrode

Thomas Wischgoll

Todd Yeates

Tau-Mu Yi

Ahmet Yildiz

Kai Ying

Kevin Yuk-Lap Yip

Menno Witter

Judith Wodke

Florentin Woergoetter

Shibu Yooseph

Darrin York

Lingchong You

Charles Wolgemuth

Peter Young

Thilo Womelsdorf

Alistair Young

Chung Wong

Nada Yousif

James Wood

Rolf Ypma

Michael Woodhams

Kim Worley

Michael Worobey

Pengfei $Y u$

Haibo $\mathrm{Yu}$

Haiyuan Yu 


\section{PLOS Compurational}

Kebing Yu

Nadine Ziemert

Guo-Cheng Yuan

Ralf Zimmer

Karina Yusim

Christophe Zimmer

Ulrich Zachariae

Andrei Zinovyev

Bojan Zagrovic

Qasim Zaidi

Alexey Zaikin

Andrew Zalesky

Naif Zaman

Roya Zandi

Bas-Jan Zandt

Ronen Zangi

Sara Zanivan

Noam Ziv

Michal Zochowski

Ali Zomorrodi

Jinfeng Zou

Jun Zou

Paolo Zunino

Mark Zwart

Igor Zwir

Jeremiah Zartman

Joel Zylberberg

Maria Zavodszky

Guenther Zeck

Peter Zee

Jonathan Zelner

Friedemann Zenke

Chang-Guo Zhan

Xuegong Zhang

Sulin Zhang

Lei Zhang

Chi Zhang

Jianzhi Zhang

Jing Zhang

Yu Zhang

Xian Sheng Zhang

Henggui Zhang

Xiaoyan Zhang

Le Zhang

Kechen Zhang

Shihua Zhang

Yuping Zhang

Shunan Zhang

Haiqing Zhao

Ting Zhao

Lili Zhao

Fangqing Zhao

Yi Zhao

Jie Zheng

Heping Zheng

Wenjun Zheng

Yujun Zheng

Deyou Zheng

Sheng Zhong

Joseph Zhou

Huan-Xiang Zhou

Yaogi Zhou

Xiaobo Zhou

Baiyu Zhou

Changsong Zhou

Fangqiang Zhu

Victor Zhurkin

Piotr Zielenkiewicz 